\title{
EDITORIAL
}

\section{Sexual dimorphism in response to stress}

\author{
Rubén Retuerto ${ }^{1}$, Julia Sánchez Vilas², Sandra Varga ${ }^{3}$ \\ ${ }^{1}$ Universidade de Santiago de Compostela, Department of Functional Biology, Lope Gómez \\ de Marzoa s/n, 15782 Santiago de Compostela (Spain) ruben.retuerto@usc.es \\ ${ }^{2}$ Cardiff University, Organisms and Environment Division, Cardiff School of Biosciences, \\ The Sir Martin Evans Building, Museum Avenue, Cardiff, CF10 3AX (UK) \\ sanchezvilasj@cradiff.ac.uk
}

\begin{abstract}
${ }^{3}$ University of Lincoln, College of Science, School of Life Sciences, Joseph Banks Laboratories, Green Lane, Lincoln, LN6 7TS (UK) svarga@lincoln.ac.uk
\end{abstract}

Sexual dimorphism in secondary traits (differences between the sexes in characteristics others than the sexual organs) is widespread in plants (Geber and Dawson, 1999; Barrett and Hough 2013). Sex differences in ecological, morphological and physiological traits have been commonly attributed to the different costs of reproduction associated with the male and the female function (Reznick, 1985; Obeso, 2002) and are usually linked to trade-offs between allocation to reproduction and to other plant functions (e.g., to growth and/or defence). Such trade-offs are likely to be modified under more stressful conditions (Bazzaz and Grace, 1997). In fact, plants are continuously exposed to stressful biotic and abiotic environmental factors during all their life cycle, which usually occur simultaneously (Suzuki et al., 2014). Biotic factors include competitors, symbionts, parasites, pathogens, and herbivores. Abiotic factors include extreme temperatures, water, light and nutrient availability, heavy metals, $\mathrm{CO}_{2}$, or UV radiation. As sessile organisms, plants have developed a wide range of responses to cope with stress, ranging from escape to tolerance and avoidance (Lerner, 1999).

Understanding how environmental stress affects plant communities is especially important in plants with sexually dimorphic breeding systems, as the sexes usually experience the environmental pressures in a different way. Because the female function is generally associated with a greater demand for resources, needed to produce both flowers and seeds, female plants are usually assumed to perform worse than males under stress and malebiased sex ratios are commonly found in more stressful environments (Barrett et al., 2010). Importantly, highly skewed sex ratios as a consequence of sex specific responses could lead to population declines and even extinction with potential effects on the structure and stability of communities, which has been very rarely studied (Munné-Bosch, 2015; Hultine et al., 2016; Petry et al., 2016). Likewise, sex-specific differences in stress tolerance and habitatrelated differences in performance put dimorphic species especially at risk due to the rapid environmental changes underway. It is urgent to resolve uncertainties in order to increase our 
ability to predict the extent to which the on-going environmental changes may impact dimorphic species. The goal of this special issue was to expand our knowledge on sexspecific responses to environmental stress in plants.

In the opening paper of this issue, Charlesworth (2017) highlights dioecious species as interesting systems for studying the evolution of sexual dimorphism. Once separate sexes evolve from hermaphroditism, adaptive divergence in traits of males and females can be expected as the result of sex-specific selection pressures associated with their reproductive function (Barrett and Hough, 2013). However, there are still many unresolved issues concerning how and why trait differences between the sexes have evolved. Here, Charlesworth (2017) discusses approaches for obtaining evidence for the occurrence of adaptation after dioecy has evolved, and to what extent adaptive changes are sexually antagonistic (having beneficial effects on one sex, but detrimental in the opposite sex). Charlesworth (2017) suggests a number of interesting questions for future empirical work on dioecious plants aimed at increasing our understanding of the genetics and evolution of sexual dimorphism.

Combined with previous studies, evidence suggests that there is not yet a clear pattern in the responses of the sexes to environmental stresses, and their differences seem to vary depending on the species considered and the type of stress, demanding a greater range of studies that allow generalisations (Juvany and Munné-Bosch, 2015). As emphasised by Suzuki et al. (2014), recent studies have shown that the response of plants to multiple simultaneous stresses cannot be extracted from the response to individual stress, highlighting the need for more and larger studies encompassing multiple stress factors. In this Special Issue, Strømme et al. (2017) and Canelles et al. (2017) consider this integrative approach and incorporate climatic gradients into their studies. Global warming and associated climate change are predicted to greatly influence the levels of Ultraviolet -B (UV-B) radiation at Earth's surface (Williamson et al., 2014). Strømme et al. (2017) consider the extent to which the interaction between UV-B radiation and temperature, two factors rarely studied together, can yield divergent responses between the sexes in the dioecious tree Populus tremula. They study male and female plants grown along a natural temperature and UV-B gradient in central Norway; reducing UV-B using specific screening filters and measuring effects on growth, timing of terminal bud formation and bud break, carbon and nitrogen content and concentrations of phenolic compounds. They found that elevation and UV-B radiation affected concentrations of nitrogen and phenolics in stems and leaves. They also found that increased elevation had a negative effect on plant growth and promoted autumnal bud formation; but most importantly, the magnitude and direction of sexual differences depended on elevation. Moreover, males were more sensitive to the effects of UV-B, with delayed bud formation and enhanced growth at the highest elevation under the UV-B attenuation treatment.

The relationship among reproduction patterns, floral dimorphism and environmental stress is considered across a climatic gradient by Canelles et al. (2017). In their integrative study on Silene acaulis, a widespread gynodioecious plant of boreo-alpine ecosystems, they 
found that females showed larger ovary volumes and longer styles, but smaller corolla widths and petal length than hermaphrodites. However, plant size and flower number per plant did not differ significantly between sexes, although flower production was positively related to plant size in both sexes and to fruit production in females, but not in hermaphrodites. The study reveals a strategy in females to compensate for differences in reproductive investment, and thus for the maintenance of gynodioecy, through higher fruiting production and other components of fitness than hermaphrodites. Unexpectedly, environment severity was not related to most of the biological traits studied, and only germination success was negatively correlated with suitability index, regardless of sex. The authors suggest that the low overall influence of climatic suitability in explaining differences in production may respond to particularities of each population (e.g. interspecific competition, soil quality, pollinators, etc.), and to the longevity of $S$. acaulis, which can attain well over 100 years. The hypothesis that hermaphrodites could lose their female function in more severe climatic environments, and therefore that gynodioecy may decline in favour of dioecy, was not clearly supported by their results, although the authors claimed that more studies considering pollen quantity and quality are needed to test this hypothesis.

The impact of climate change on performance of the sexes in the dioecious tree Populus tremula is also the focus of the paper by Sobuj et al. (2017). They investigated the single and combined effects of elevated temperature and $\mathrm{CO}_{2}$ concentration on the growth and carbon-based secondary metabolites synthesis in stem bark of male and female plantlets. They observed that overall height growth in males was greater than in females. However, under elevated temperature, height growth and diameter were considerably greater in females than in males. From this result, the authors infer variation in optimum growth temperature between females and males in P. tremula. Contrary to expectations that elevated $\mathrm{CO}_{2}$ will have no effect on growth, since the initial stimulation of $\mathrm{CO}_{2}$ is not usually sustained through time in broad-leaved trees (Vu et al., 2002), diameter incremented more in females than in males in response to $\mathrm{CO}_{2}$. However, the authors did not find effects of sex on biomass, suggesting that the absence of a cost of reproduction in $P$. tremula seedlings might explain the smaller intersexual differences in growth. Flavonoids, phenolic acids and salicylates are important compounds in protecting plants against a wide range biotic and abiotic stress (Lindroth and Clair, 2013; Julkunen-Tiitto et al., 2015). In line with this, Sobuj et al. (2017) suggest that increased concentrations of these metabolites may provide a potential fitness advantage in the face of environmental changes. In this respect, they found that females had a higher total concentration of flavonoids in comparison with males. However, under elevated $\mathrm{CO}_{2}$, females reduced the synthesis whereas it was increased in males. Regarding phenolic acids, the total concentration was greater in females than in males. In both sexes, the synthesis of phenolic acids was reduced under elevated temperature, but it was increased under elevated $\mathrm{CO}_{2}$. The same trend was observed for salicylates, except that under elevated $\mathrm{CO}_{2}$ the excess synthesis only occurred in male plants. Overall, males tended to have higher growth, while females tended to have greater concentrations of phenolics. This study concludes that under scenarios with concomitant increases in $\mathrm{CO}_{2}$ and temperature, the sexes of $P$. tremula will probably grow more and accumulate lower levels of phenolics, although the reported effects may be more pronounced in sexually mature plants. These three studies 
highlight once more how the response of the sexes may be context-dependent and the need to carry out further research including multiple interacting factors.

The profound effect that environmental stress can have on sex ratios in dioecious plant populations is highlighted in the paper by Simancas et al. (2017). Whilst sex-lability is common in plant species, the mechanisms underlying changes in sex expression are not well understood. In their work, they found that when dioecious Urtica dioica plants were exposed to phosphate starvation, males delayed their transition to flowering, and the sex ratio of the experimental population shifted towards females. Seed production may be negatively affected by this stress, and they hypothesised that the observed shift in sex ratio may serve as a mechanism to compensate for seed production at the population level. In addition, their experiment involved hormonal profiling that unravelled differences between the sexes in response to phosphate starvation. In particular, they found that this stress increased the level of compounds related to herbivory defence only in females. Stressful environmental conditions may modify not only the number of flowers produced but also the sexual expression of these flowers. In a contribution by Buide et al. (2017) sexual plasticity in gynodioecious-gynomonoecious Silene littorea was demonstrated, with a reduction in the total amount of flowers produced in shaded plants but an increase in the proportion of female flowers. Even though the physiological mechanisms were not investigated, plant hormones together with resource availability are the probable causes.

Biased-sex ratios may impact, and be impacted, by interactions with other organisms aboveground. Moritz et al. (2017) investigated the relatively well-established sex-biased herbivory preference in dioecious willows using a large-scale approach. The authors created experimental stands mimicking commercial plantation designs that contained either females, males or even-sex mixes, and monitored herbivory damage by roe deer after one growth season. Their results showed that mixed-sex plots were more preferred over mono-sex plots, however they could not demonstrate an individual preference for one sex within mixed-sex plots. The authors attributed these results to the differences in secondary metabolite profiles between the sexes rather than the total content of secondary metabolites or nutrients. Studies like this highlight the need for large-scale manipulative field experiments to evaluate herbivory choice and to understand the mechanisms and consequences behind sex-biased herbivory.

While the previous studies highlight the evolutionary forces driving sexual dimorphism in response to stress, extra efforts should be directed towards elucidating the molecular and physiological mechanisms underlying sex-specific responses. For example, previous studies have reported that sexual dimorphic plants may have sex-specific adaptations to heavy metal contamination, with most of these studies showing that males exhibited a greater adaptive capacity than females when exposed to heavy metal stress (e.g., Chen et al., 2011; Han et al., 2013, but see Sanchez Vilas et al., 2016). In this Special Issue, Qin et al. (2017) hypothesised that application of zinc, a metal that acts as a signal to mediate the response to oxidative stress, could reduce lead-induced stress more in female individuals than in males. To test this hypothesis, they examined sex differences in morphological 
characteristics, biomass, gas exchange, cell ultrastructure and antioxidant enzyme activity in saplings of a dioecious tree species widely planted as an important economic species, the mulberry (Morus alba). Their study shows that sexes of mulberry saplings differed in morphological traits, growth, gas exchange, enzyme activity, electrolyte leakage and cellular ultrastructure in response to lead and zinc. Overall, lead stress negatively affected females more than males, suggesting that males could have better detoxification mechanisms and adaptation strategies in response to lead stress, and consequently, to be a better candidate for phytoremediation. The application of zinc improves lead tolerance in both sexes but, as hypothesised, the effect was more pronounced in females than in males

We hope that the papers in this Special Issue motivate further research on the study of sexual dimorphism in response to environmental stress. Given the increasing concerns over the effects of global change on biological communities, there is a pressing need for further research on the mechanisms underlying adaptive responses of plants to environmental changes. Dioecious species are ideal comparative systems in which to investigate adaptation, since they allow us to examine the functional significance of sexual dimorphism without the confounding effects of differences in geography, history and ecology (Dawson and Bliss, 1989; Dawson and Geber, 1999). The rapid global changes underway will provide new chances to study sex differences in adaptation. Changes in the climate will surely induce sexspecific adjustments in the patterns of distribution of sexually dimorphic species, which will move to new environments, disrupting their physiology, phenology and reproduction, as testified by four of the papers published in this Special Issue.

An understanding of the basic mechanistic factors that underlay the responses of plants to multiple stresses should benefit from integrative studies across multiple levels of biological organisation, analysing transcriptional, metabolomic and physiological data. Plant biology has become an increasingly data-rich field of research fuelled by the rapid advances in analytical techniques. However, although the use of the "-omics" approaches (i.e.: genomic, proteomic, metabolomic) could provide novel insights into the proximate mechanisms responsible for sexual divergence in the responses to stress, they are still used insufficiently in this field of research. Fortunately, five of the papers that appear in this Special Issue follow this approach and use some of these promising tools for research on sexrelated differences in dioecious plants in response to stress.

The lack of general patterns emerging from the studies in this Special Issue are certainly related to species-specific responses to stress and to the different nature (biotic and abiotic) and intensities of the stresses studied, which could differentially modify the tradeoffs between allocation to reproduction and to other plant functions. Differences in the reproductive status of the experimental plants (four studies in this Special Issue considered pre-reproductive plants) could also explain the inconsistencies found, since intersexual differences in growth, reproduction and physiology may be more pronounced after sexual maturation (Dawson and Geber, 1999). Because sexes may differ in the timing of key developmental stages (e.g., germination, flowering, senescence) (Lloyd and Webb, 1977) and in the frequency and intensity of reproductive events, measurements at a single point in time 
may not realistically reflect time-integrated patterns (Sanchez Vilas et al., 2012). Future research would benefit from examining long-term differences in addition to short-term ones for a thorough understanding of the quantitative relationships between male and female reproductive efforts over the whole growing season, or even over the whole life-cycle of perennial plants. Lastly, little is known on patterns of dimorphism in below-ground processes and structures. Considering that sexual dimorphism would be the outcome of below versus above processes (Varga et al., 2017), and allocation to above and below-ground sinks (Harris and Pannell, 2008), studies that will focus on these aspects would be especially useful to make a compelling story of sexual dimorphism in plants under stress.

\section{ACKNOWLEDGEMENTS}

We are grateful to Sergi Munné-Bosch for inviting us to guest edit this Special Issue and for his help and support throughout. We are also indebted to the authors for their contributions and to the many reviewers for their helpful constructive comments on the manuscripts. Thanks also to John Pannell and Sergi Munné-Bosch for their comments on this manuscript.

\section{References}

Barrett, S. C. H., Hough, J., 2013. Sexual dimorphism in flowering plants. J. Exp. Bot. 64, 67-82.

Barrett, S.C.H., Yakimowski, S.B., Field, D.L., Pickup, M., 2010. Ecological genetics of sex ratios in plant populations. Phil. Trans. R. Soc. B. 365, $2549 \mathrm{e} 2557$.

Bazzaz, F. A., Grace, J., 1997. Plant Resource Allocation. Academic Press.

Buide, M. L., del Valle, J. C., Castilla, A. R., Narbona, E., 2017. Sex expression variation in response to shade in gynodioecious-gynomonoecious species: Silene littorea decreases flower production and increases female flower proportion. Env. Exp. Bot. $\mathrm{XX}, \mathrm{XX}-\mathrm{XX}$.

Canelles, Q., Saura-Mas, S., Brotons, L., García, M. B., Lloret, F., Vilellas, J., Morris, W. F., 2017. Environmental stress effects on reproduction and sexual dimorphism in the gynodioecious species Silene acaulis. Env. Exp. Bot. XX, XX-XX.

Charlesworth, D., 2017. XXX. Env. Exp. Bot. XX, XX-XX.

Chen, L., Han, Y., Jiang, H., Korpelainen, H., Li, C., 2011. Nitrogen nutrient status induces sexual differences in responses to cadmium in Populus yunnanensis. J. Exp. Bot. 62, 5037-5050.

Dawson, T.E., Bliss, L.C., 1989. Patterns of water use and the tissue water relations in the dioecious shrub, Salix arctica: the physiological basis for habitat partitioning between the sexes. Oecologia 79, 332-343.

Dawson, T.E., Geber, M.A., 1999. Sexual dimorphism in physiology and morphology. In: Geber, M.A., Dawson, T.E., Delph, L.F. (Eds.), Gender and Sexual Dimorphism in Flowering Plants. Springer, pp. 175-215. 
Geber, M.A., Dawson, T E., Delph, L.F., 1999. Gender and Sexual Dimorphism in Flowering Plants. Springer.

Han, Y., Wang, L., Zhang, X., Korpelainen, H., Li, C., 2013. Sexual differences in photosynthetic activity, ultrastructure and phytoremediation potential of Populus cathayana exposed to lead and drought. Tree Physiol. 33, 1043-1060.

Harris, M.S:, Pannell, J.R., 2008. Roots, shoots and reproduction: sexual dimorphism in size and costs of reproductive allocation in an annual herb. Proc. R. Soc. B. 275, 25952602.

Hultine, K.R., Grady, K C., Wood, T.E., Shuster, S.M., Stella, J.C., 2016. Climate change perils for dioecious plant species. Nature Plants, 16109.

Julkunen-Tiitto, R., Nenadis, N., Neugart, S., Robson, M., Agati, G., Vepsäläinen, J., Zipoli, G., Nybakken, L., Winkler, B., Jansen, M.A.K., 2015. Assessing the response of plant flavonoids to UV radiation: an overview of appropriate techniques. Phytochem. Rev. 14, 273-297.

Juvany, M., Munné-Bosch, S., 2015. Sex-related differences in stress tolerance in dioecious plants: a critical appraisal in a physiological context. J. Exp. Bot. 66, 6083-6092.

Lerner, H.R., 1999. Plant Responses to Environmental Stresses: from phytohormones to genome reorganization. CRC Press.

Lindroth, R.L., Clair, S.B.S., 2013. Adaptations of quaking aspen (Populus tremuloides Michx.) for defense against herbivores. For. Ecol. Manage. 299, 14-21.

Lloyd, D.G., Webb, C.J., 1977. Secondary sex characters in plants. Bot. Review 43, 177-216.

Moritz, K. K., Parachnowitsch, A. L., Julkunen-Tiitto, R., Björkman, C., Ayres, M. P., Stenberg, J. A., 2017. Roe deer prefer mixed-sex willow stands over monosexual stands but do not discriminate between male and female plants. Env. Exp. Bot. XX, $\mathrm{XX}-\mathrm{XX}$.

Munné-Bosch, S., 2015. Sex ratios in dioecious plants in the framework of global change. Env. Exp. Bot.109, 99-102.

Obeso, J.-R., 2002. The costs of reproduction in plants. New Phytol. 155, 321-348.

Petry, W.K., Soule, J.D., Iler, A.M., Chicas-Mosier, A., Inouye, D.W., Miller, T.E.X., Mooney, K.A., 2016. Sex-specific responses to climate change in plants alter population sex ratio and performance. Science 353, 69-71.

Qin, F., Liu, G., Huang, G., Dong, T., Liao, Y., Xu, X., 2017. Zinc application alleviates the adverse effects of lead stress more in female Morus alba than in males. Env. Exp. Bot. XX, XX-XX.

Reznick, D., 1985. Costs of reproduction: an evaluation of the empirical evidence. Oikos 44, 257-267.

Sánchez Vilas, J., Bermúdez, R., Retuerto, R., 2012. Soil water content and patterns of allocation to below- and above-ground biomass in the sexes of the subdioecious plant Honckenya peploides. Ann. Bot. 110, 839-848.

Sanchez Vilas, J., Campoy, J.G., Retuerto, R., 2016. Sex and heavy metals: Study of sexual dimorphism in response to soil pollution. Env. Exp Bot. 126, 68-75.

Simancas B, Cotado A, Müller M, Munné-Bosch S., 2017. Phosphate starvation during the transition phase increases the sex ratio and 12-oxo-phytodienoic acid contents in females of Urtica dioica. Env. Exp. Bot. 145, 39-46. 
Sobuj, N., Virjamo, V., Zhang, Y., Nybakken, L., Julkunen-Tiitto, R., 2017. Impacts of elevated temperature and $\mathrm{CO}_{2}$ concentration on growth and phenolics in the sexually dirmophic Populus tremula (L.). Env. Exp. Bot. XX, XX-XX.

Strømme, C. B., Julkunen-Tiito, R., Olsen, J. E., Nybakken, L. 2017. The dioecious Populus tremula displays interactive effects of temperature and ultraviolet-B along a natural gradient. Env. Exp. Bot. XX, XX-XX.

Suzuki, N., Rivero, R.M., Shulaev, V., Blumwald, R.M., 2014. Abiotic and biotic stress combinations. New Phytol. 203, 32-43.

Varga, S., Vega-Frutis, R., Kytoviita, M.M., 2017. Competitive interactions are mediated in a sex-specific manner by arbuscular mycorrhiza in Antennaria dioica. Plant Biol. 19, 217-226.

Vu, J.C.V., Newman, Y.C., Allen, L.H., Gallo-Meagher, M., Zhang, M.Q., 2002.

Photosynthetic acclimation of young sweet orange trees to elevated growth $\mathrm{CO}_{2}$ and temperature. J. Plant Physiol. 159, 147-157.

Williamson, C.E., Zepp, R.G., Lucas, R.M., Madronich, S., Austin, A.T., Ballaré, C.L., Norval, M., Sulzberger, B., Bais, A.F., McKenzie, R.L., Robinson, S.A., Häder, D.-P., Paul, N.D., Bornman, J.F., 2014. Solar ultraviolet radiation in a changing climate. Nat. Clim. Chang. 4, 434-441. 